Acta Crystallographica Section F

Structural Biology

and Crystallization

Communications

ISSN 1744-3091

Geoffrey K.-W. Kong, ${ }^{a, b}$ Denise Galatis, $^{\text {b,c }}$ Kevin J. Barnham, ${ }^{\text {b,c }}$

Galina Polekhina, ${ }^{a}$ Julian J.

Adams, ${ }^{a}$ Colin L. Masters, ${ }^{b, c}$

Roberto Cappai, ${ }^{b, c, d}$ Michael W.

Parker $^{\mathrm{a}}$ and William J.

McKinstry $^{\mathrm{a} *}$

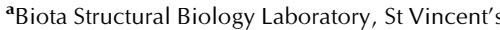
Institute, 9 Princes Street, Fitzroy, Victoria 3065, Australia, ${ }^{\mathbf{b}}$ Department of Pathology, The University of Melbourne, Victoria 3010,

Australia, ' $T$ The Mental Health Research Institute of Victoria, Parkville, Victoria 3052, Australia, and ${ }^{\mathbf{d}}$ Centre for Neuroscience, The University of Melbourne, Victoria 3010, Australia

Correspondence e-mail: wmckinstry@svi.edu.au

Received 7 September 2004 Accepted 15 November 2004

Online 2 December 2004

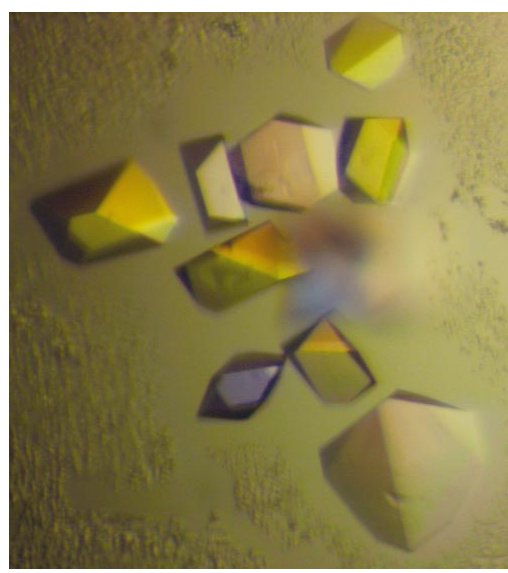

(C) 2005 International Union of Crystallography All rights reserved

\section{Crystallization and preliminary crystallographic studies of the copper-binding domain of the amyloid precursor protein of Alzheimer's disease}

Alzheimer's disease is thought to be triggered by production of the amyloid $\beta$ $(\mathrm{A} \beta)$ peptide through proteolytic cleavage of the amyloid precursor protein (APP). The binding of $\mathrm{Cu}^{2+}$ to the copper-binding domain (CuBD) of APP reduces the production of $\mathrm{A} \beta$ in cell-culture and animal studies. It is expected that structural studies of the $\mathrm{CuBD}$ will lead to a better understanding of how copper binding causes $\mathrm{A} \beta$ depletion and will define a potential drug target. The crystallization of $\mathrm{CuBD}$ in two different forms suitable for structure determination is reported here.

\section{Introduction}

Alzheimer's disease is a debilitating disorder confronting ageing populations worldwide. It is increasingly accepted that Alzheimer's disease is brought about by an increased production of the amyloid $\beta$ $(\mathrm{A} \beta)$ peptide, which is neurotoxic and compromises neuronal function (Selkoe, 2002). The peptide arises through the cleavage of the amyloid precursor protein (APP; Kang et al., 1987) by the sequential cleavage of $\beta$-site APP-cleaving enzyme (BACE) and the protein complex $\gamma$-secretase. APP is a type I transmembrane protein with a large extracellular portion and may play a role in signalling (see, for example, Cao \& Sudhof, 2001) or interaction with the extracellular matrix (Rossjohn et al., 1999).

APP is able to bind $\mathrm{Cu}$ ions (Hesse et al., 1994) and can modulate the level of copper ions in the brain by transporting the ions in and out of neurones (White et al., 1999; Maynard et al., 2002; Bellingham et al., 2004). The binding of $\mathrm{Cu}^{2+}$ ions to the extracellular copperbinding domain $(\mathrm{CuBD})$ can lower the production of $\mathrm{A} \beta$ in cultured cells (Borchardt et al., 1999). The beneficial effects of $\mathrm{Cu}^{2+}$ binding to the APP have been demonstrated recently in two independent transgenic mouse studies. By supplementing the drinking water with $\mathrm{Cu}^{2+}$, the survival of APP23 mice (carrying a mutant APP that overproduces $\mathrm{A} \beta$ ) was improved, while there was increased bioavailable $\mathrm{Cu}^{2+}$ and reduced $\mathrm{A} \beta$ in the brain (Bayer et al., 2003). Similar effects were achieved by crossing TgCRND8 mice with mice that have raised brain $\mathrm{Cu}$ levels owing to the expression of a mutant copper transporter CuATPase $7 \mathrm{~b}$. This resulted in decreased amyloid plaque and plasma A $\beta$ levels (Phinney et al., 2003). Structural studies of $\mathrm{Cu}^{2+}$ binding to APP will thus aid the discovery of drugs that mimic the beneficial effects of $\mathrm{Cu}^{2+}$ in and may lead to a treatment for Alzheimer's disease.

The structure of the apo form of the CuBD has been determined using NMR spectroscopy (Barnham et al., 2003). The structure was found to consist of an $\alpha$-helix packed against a three-strand $\beta$-sheet, a fold also observed in several copper chaperones. Copper-binding residues were inferred by titration of $\mathrm{Cu}^{2+}$ ions into a solution of CuBD. The resonance peaks of His147, His151, Tyr168 and Met170 were broadened owing to the paramagnetic interference of $\mathrm{Cu}^{2+}$. A crystallographic study of the $\mathrm{CuBD}$ has been pursued to confirm these residues as ligands, to study the binding site in detail and to understand the copper-reduction mechanism. 


\section{Experimental procedures}

\subsection{Expression and purification of APP133-189 in Pichia pastoris}

The copper-binding domain of APP (residues 133-189) was amplified from APP695 cDNA using the primers CuBDforward, GGTCGACAAAAGAGAGGCTTGCAAATTCTTACACCAGG, and CuBDreverse, GAATTCTTACAGTGGGCAACACACAAACTC. The PCR DNA was cloned as a SalI-EcoRI fragment into XhoI-EcoRI-digested pPIC-9 expression vector (Invitrogen). The pIC9-APP133-189 vector was linearized with BglII and transfected into P. pastoris strain GS115 as previously described (Henry et al., 1997). A strongly expressing clone was selected by screening culture supernatants by silver staining of SDS-PAGE gels. Expression cultures were grown for $48 \mathrm{~h}$ in YPD medium and induced in YP medium containing 3\%(v/v) methanol for $24 \mathrm{~h}$. Cells were removed by centrifugation $(10000 \mathrm{~g}, 277 \mathrm{~K}, 30 \mathrm{~min})$ and the medium passed through a $0.2 \mu \mathrm{m}$ filter. The protein was concentrated to $10 \mathrm{ml}$ or less using a stirred-cell concentration device. The concentrated sample was then purified using a Superdex-75 gel-filtration column (Pharmacia Biotech, UK) and eluted with $20 \mathrm{~m} M$ MES pH 6.8. The peak corresponding to $\mathrm{CuBD}$, as determined by SDS-PAGE, was concentrated using a Vivaspin-2 (3000 Da molecular-weight cutoff; VivaScience, Germany) to a final concentration of $\sim 10 \mathrm{mg} \mathrm{ml}^{-1}$. The metal-ion content of the protein was analysed by inductively coupled plasma mass spectrometry (ICP-MS).

\subsection{Crystallization}

Crystallization trials were carried out at $295 \mathrm{~K}$ using the hangingdrop method by mixing 1-2 $\mu$ protein solution with an equal volume of the reservoir. The initial crystallization conditions were established using the protein crystallization screen devised by Zeelen et al. (1994) and Crystal Screen II (Hampton Research, USA). After further optimization and streak-seeding with older crystals using cat whiskers, crystals of good diffraction quality could be obtained. Crystal soaking in the presence of $\mathrm{Cu}^{2+}$ is currently being attempted.

\subsection{Data collection and processing}

The diffraction data from a type A crystal (see $\S 3$ ) were collected at Sector 14-BM-C of the Advanced Photon Source synchrotron (Argonne, Illinois, USA) at $100 \mathrm{~K}$. Data from a type B crystal were collected at $115 \mathrm{~K}$ using an in-house MAR Research area detector with $\mathrm{Cu} K \alpha \mathrm{X}$-rays generated by a Rigaku RU-200 rotating-anode $\mathrm{X}$-ray generator. The crystals were cryoprotected in their respective reservoir buffers supplemented with $12-14 \%(v / v)$ glycerol. The data were processed using the $H K L$ suite of programs (Otwinowski, 1993) and converted into $\mathrm{CCP} 4$ format (Collaborative Computational Project, Number 4, 1994). Molecular replacement was attempted using the NMR structure (PDB code 1owt; Barnham et al., 2003) as a probe model in the CCP4 programs MOLREP (Vagin \& Teplyakov, 1997) and AMoRe (Navaza, 1994).

\section{Results and discussion}

The domain boundaries of the copper-binding domain (CuBD) were chosen so that the N-terminus (Ser124) followed immediately from the N-terminal growth-factor domain (Rossjohn et al., 1999) and the C-terminus (Leu189) fell immediately before the start of a region rich in acidic residues. Initial attempts to crystallize CuBD124-189 resulted in very small needle crystals that could not be optimized despite extensive attempts. We then decided to pursue structure determination using NMR spectroscopy (Barnham et al., 2003). We
Table 1

Data-collection statistics for crystals of types A and B.

Values in parentheses indicate data in the highest resolution shell (1.66-1.60 $\AA$ for type A, $1.67-1.61 \AA$ for type B).

\begin{tabular}{lll}
\hline Crystal & Type A & Type B \\
\hline Maximal resolution $(\AA)$ & 1.60 & 1.61 \\
Space group & $P 2_{1} 2_{1} 2_{1}$ & $P 2_{1} 2_{1} 2_{1}$ \\
Unit-cell parameters & 61.4 & 31.4 \\
$\quad a(\AA)$ & 68.0 & 32.5 \\
$b(\AA)$ & 127.8 & 50.2 \\
$\quad c(\AA)$ & $11.7(3.4)$ & $2.72(2.20)$ \\
Redundancy & $88.2(58.9)$ & $96.2(89.8)$ \\
Completeness $(\%)$ & $85.3(46.9)$ & $86.6(65.7)$ \\
Completeness $>2 \sigma(\%)$ & $25.6(1.24)$ & $15.5(5.39)$ \\
$I / \sigma(I)$ & $8.2(67.1)$ & $5.8(17.8)$ \\
$R_{\text {merge }}(\%)$ &
\end{tabular}

successfully solved the NMR structure of apo APP124-189, but could not visualize the $\mathrm{Cu}^{2+}$-bound form of $\mathrm{CuBD}$ owing to the paramagnetism of $\mathrm{Cu}^{2+}$, which led to severe line-broadening of the spectra. Importantly, the NMR structure revealed a flexible $\mathrm{N}$-terminal region spanning residues $124-132$, which we reasoned might impair crystallization. We therefore expressed another construct in which the flexible N-terminal residues 124-132 were removed. The APP133-189 construct was expressed in P. pastoris,

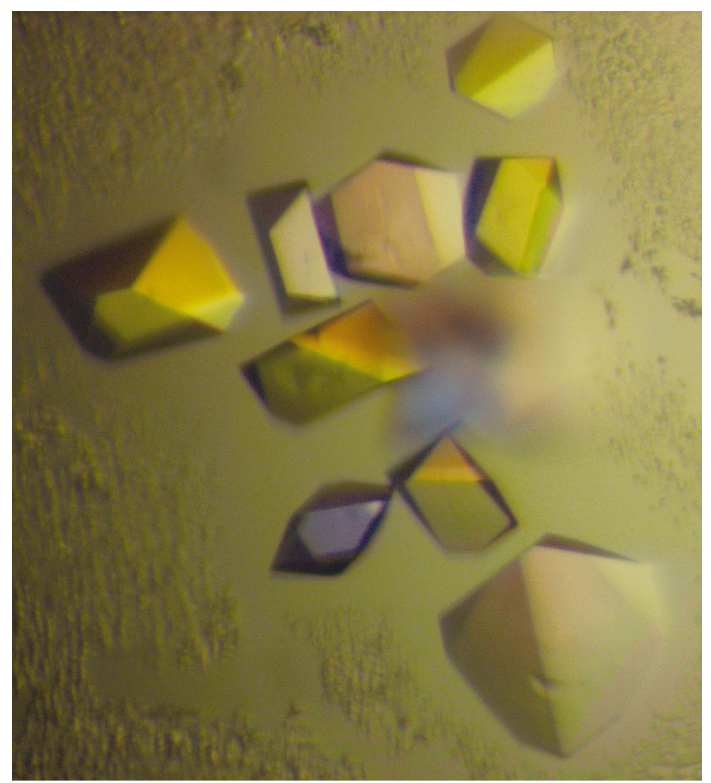

(a)

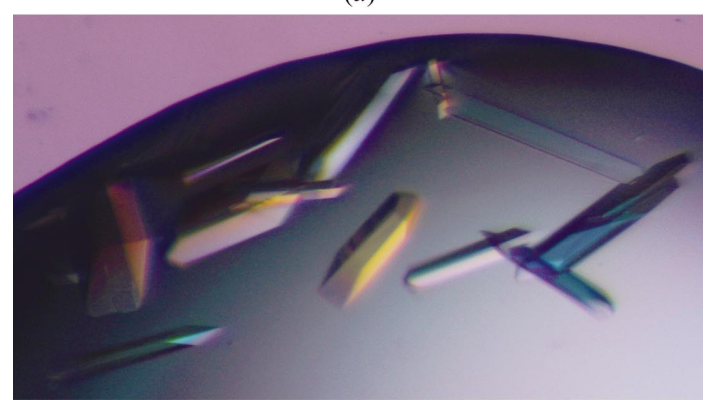

(b)

Figure 1

Crystals of CuBD grown in (a) $0.1 M$ MES pH 5.5, $0.4 M \mathrm{NaCOOH}$ plus 10 $15 \%(w / v)$ PEG 4000 and $(b) 0.1 M$ HEPES pH 8.0 plus $28-32 \%(w / v)$ PEG. The longest dimension of a typical crystal grown under both conditions is between 150 and $200 \mathrm{~mm}$. 


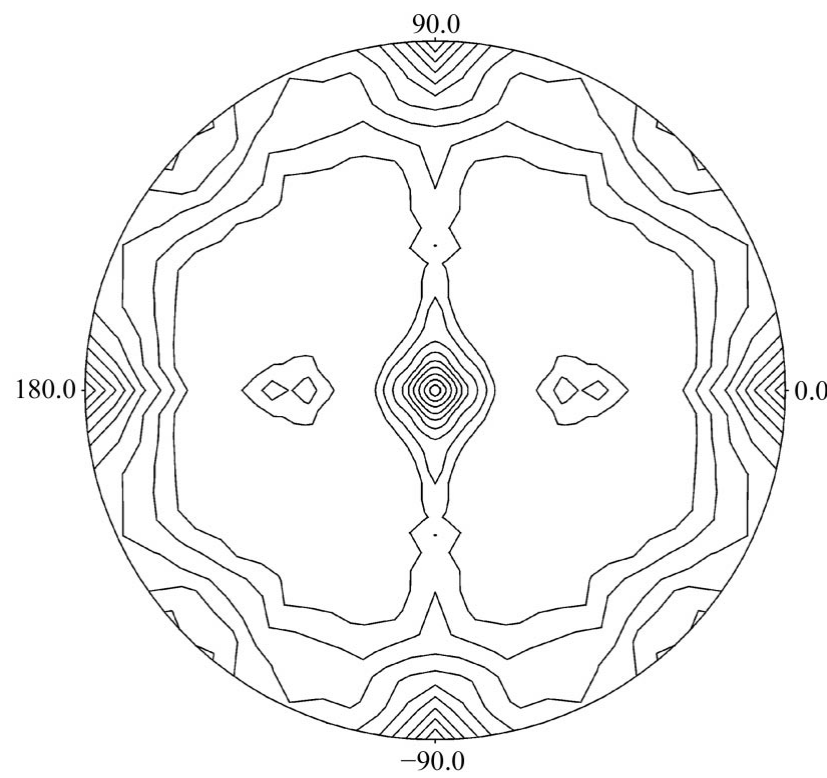

Figure 2

Self-rotation function calculated from diffraction data of type A crystals at $\kappa=180^{\circ}$, using data between 20.0 and $4.0 \AA$ resolution and the program POLARRFN (Collaborative Computational Project, Number 4, 1994). The horizontal and vertical polar coordinates of the rotation axis are $\omega\left(0-90^{\circ}\right.$ from centre to edge of circle) and $\varphi$ (plotted around the circumference as shown in the figure). The peaks at $\varphi=0,90,180$ and $-90^{\circ}$ on the circumference arise from crystallographic twofold of the space group.

with an expression level of 10-20 mg purified protein per litre. ICPMS analysis of the purified protein showed insignificant amounts of copper ions.

Two crystallization conditions were discovered from each of the two crystallization screens used. Type A crystals were the first to appear. They were grown in 0.1 $M$ MES pH 5.5, 0.4 M NaCOOH plus $10-15 \%(w / v)$ PEG 4000 and usually reached $100-150 \mu \mathrm{m}$ in each dimension (Fig. 1). They diffracted to a maximal resolution of $1.60 \AA$ with synchrotron radiation $(\lambda=0.9 \AA)$. The data-collection statistics are given in Table 1 . The unit-cell parameters are consistent with the presence of between six and $11 \mathrm{CuBD}$ molecules per asymmetric unit using the Matthews formula $\left(V_{\mathrm{M}}=3.25\right.$ and $1.77 \AA^{3} \mathrm{Da}^{-1}$ for $n=6$ and 11, respectively; Matthews, 1968). Calculating the self-rotation function using data between 20 and $4.0 \AA$ resolution revealed complex internal symmetry; the $\kappa=180^{\circ}$ section suggested three twofold axes in 222 symmetry within each asymmetric unit (Fig. 2).

Type B crystals could be grown in $0.1 M$ HEPES $\mathrm{pH} 8.0$ plus $28-$ $32 \%(w / v)$ PEG 10000 . Crystals had the shape of rectangular prisms and reached 150-200 $\mu \mathrm{m}$ in the longest dimension (Fig. 1b). On an inhouse X-ray source, these crystals diffracted strongly to $1.61 \AA$, the physical limit of our diffractometer setup. The unit-cell parameters (see data-collection statistics in Table 1) are consistent with the presence of one CuBD in the asymmetric unit $\left(V_{\mathrm{M}}=1.94 \AA^{3} \mathrm{Da}^{-1}\right.$ with $n=1)$. Molecular-replacement calculations are in progress for both crystal forms. Trials have also been performed to introduce $\mathrm{Cu}^{2+}$ ions into the crystals through soaking. The crystals typically gained a blue-green tint and preliminary diffraction experiments with synchrotron radiation showed anomalous scattering from the $\mathrm{Cu}^{2+}$ ions.
The authors thank Irene Volitakis (The Mental Health Research Institute) for the ICP-MS analyses. GKWK is a recipient of the Australian Postgraduate Award from the Australian Government and the Ludo Frevel Scholarship from the International Centre for Diffraction Data. GP and KJB are National Health and Medical Research Council (NHMRC) R. D. Wright Fellows. WJM is an NHMRC Industry Fellow. MWP is an NHMRC Senior Principal Research Fellow. RC is an NHMRC Senior Research Fellow. This work was supported by NHMRC grants to MWP, RC, KJB and CLM and by the Australian Synchrotron Research Program, funded by the Australian Government Major National Research Facilities Program, for access to BioCARS (Sector 14) of the Advanced Photon Source (APS). Use of APS was supported by the US Department of Energy, Basic Energy Sciences, Office of Energy Research. We also thank Harry Tong and other staff at BioCARS for their help with data collection during visits to the APS.

\section{References}

Bayer, T. A., Schäfer, S., Simons, A., Kemmling, A., Kamer, T., Tepest, R., Eckert, A., Schüssel, K., Eikenberg, O., Sturchler-Pierrat, C., Abramowski, D., Staufenbiel, M. \& Multhaup, G. (2003). Proc. Natl Acad. Sci. USA, 100, 14187-14192.

Barnham, K. J., McKinstry, W. J., Multhaup, G., Galatis, D., Morton, C. J., Curtain, C. C., Williamson, N. A., White, A. R., Hinds, M. G., Norton, R. S., Beyreuther, K., Masters, C. L., Parker, M. W. \& Cappai, R. (2003). J. Biol. Chem. 278,17401-17407.

Bellingham, S. A., Ciccotosto, G. D., Needham, B. E., Fodero, L. R., White, A. R., Masters, C. L., Cappai, R. \& Camakaris, J. (2004). J. Neurochem. 91, 423-428.

Borchardt, T., Camakaris, J., Cappai, R., Masters, C. L., Beyreuther, K. \& Multhaup, G. (1999). Biochem. J. 344, 461-467.

Cao, X. \& Sudhof, T. C. (2001). Science, 293, 115-120.

Collaborative Computational Project, Number 4 (1994). Acta Cryst. D50, 760763.

Henry, A., Masters, C. L., Beyreuther, K. \& Cappai, R. (1997). Protein Expr. Purif. 10, 283-291.

Hesse, L., Beher, D., Masters, C. L. \& Multhaup, G. (1994). FEBS Lett. 349, 109-116.

Kang, J., Lemaire, H. G., Unterbeck, A., Salbaum, J. M., Masters, C. L., Grzeschik, K.-H., Multhaup, G., Beyreuther, K. \& Muller-Hill, B. (1987). Nature (London), 325, 733-736.

Matthews, B. W. (1968). J. Mol. Biol. 33, 491-497.

Maynard, C. J., Cappai, R., Volitalis, I., Cherny, R. A., White, A. R., Beyreuther, K., Masters, C. L., Bush, A. I. \& Li, Q. X. (2002). J. Biol. Chem. 277, 44670-44676.

Navaza, J. (1994). Acta Cryst. A50, 157-163.

Otwinowski, Z. (1993). Proceedings of the CCP4 Study Weekend. Data Collection and Processing, edited by L. Sawyer, N. Isaacs \& S. Bailey, pp. 5662. Warrington: Daresbury Laboratory.

Phinney, A. L., Drisaldi, B., Schmidt, S. D., Lugowski, S., Coronado, V., Liang, Y., Horne, P., Yang, J., Sekoulidis, J., Coomaraswamy, J., Cbishti, M. A., Cox, D. W., Mathews, P. M., Nixon, R. A., Carlson, G. A., St George-Hyslop, P. \& Westaway, D. (2003). Proc. Natl Acad. Sci. USA, 100, 14193-14197.

Rossjohn, J., Cappai, R., Feil, S. C., Henry, A., McKinstry, W. J., Galatis, D., Hesse, L., Multhaup, G., Beyreuther, K., Masters, C. L. \& Parker, M. W. (1999). Nature Struct. Biol. 6, 327-331.

Selkoe, D. J. (2002). Science, 298, 789-791.

Vagin, A. \& Teplyakov, A. (1997). J. Appl. Cryst. 30, 1022-1025.

White, A. R., Reyes, R., Mercer, J. F. B., Camakaris, J., Zheng, H., Bush, A. I., Multhaup, G., Beyreuther, K., Masters, C. L. \& Cappai, R. (1999). Brain Res. 842, 439-444.

Zeelen, J. P., Hiltunen, J. K., Ceska, T. A. \& Wierenga, R. K. (1994). Acta Cryst. D50, 443-447. 